\title{
Aclaraciones sobre las propuestas de adaptación terminológica al español de la estandarización de la terminología del tracto urinario inferior. El español une América Latina y España
}

\author{
Marqués Queimadelos A, Martínez García R. \\ Consenso de la SINUG y del ICCS. \\ Actas Urol Esp. 2008;32(3):271-272
}

A claraciones sobre las propuestas de adaptación terminológica al Español de la estandarización de la terminología del tracto urinario inferior. El Español une América Latina y España.

En 1988 se crea la Sociedad Iberoamericana de Neurourología y Uroginecología (SINUG) en Barcelona. La piedra base de esta sociedad era la comunicación en "Español" entre profesionales que teníamos intereses comunes: urólogos, ginecólogos, rehabilitadotes/fisiatras, neurólogos, enfermeros, psicólogos, etc. Ello hizo que pronto el objetivo de unificar conceptos y términos fuese prioritario y así, desde finales de los años ochenta, en la revista Urodinámica Aplicada, órgano de expresión de la SINUG, se han ido publicando las adaptaciones al Español de los documentos de la "ICS".

Esto, así planteado, suponía un esfuerzo de grupo. Vimos que éste era el camino y por ello se introdujo en todos los Congresos de la SINUG seminarios sobre terminología y conceptos. Los últimos se realizaron en 1996 y 2003 en Valencia, 2004 en Santiago de Compostela y 2007 en Barcelona. Se invitó a un gran número de urólogos dentro del ámbito Americano y Español con la finalidad de conseguir un documento de todos y para todos. El trabajo del grupo se presentó a los inscritos y entre ellos la representación americana fue numerosa. Cabe citar a Jara Michael de Chile, Víctor Romano de Argentina, Washington Fernández Gómez y José R. Martínez Torena de Uruguay, Paulo Palma de Brasil, Eduardo Neri y José Luis Campos de México... Los seminarios de trabajo fueron intensos y participativos durante los tres días de cada congreso.

Una vez que conseguimos un documento guía, se abrió un debate de opinión abierto en la revista
Urodinámica Aplicada. La adaptación al español se hace por consenso, no es de NADIE, es de TODOS.

Este trabajo continuado de grupo, abierto a todos ha de seguir vivo y cualquier aportación y crítica que lo mejore se agradece siempre. He de decir que, tanto la Asociación Española de Urología como la Sociedad Española de Ginecología y Obstetricia han dado el visto bueno al planteamiento.

En 2006, la Sociedad Española de Ginecología y Obstetricia (SEGO) y la Asociación Española de Urología (AEU) dan soporte al "tratado de Incontinencia Urinaria" cuyos editores son la Dra. Montserrat Espuña Pons y el Dr. David Castro Díaz y en el que se presenta en su primer capítulo el trabajo de actas de 2005 .

Me sorprende que apareciera en la Revista Chilena de Urología Volumen $71 / \mathrm{n}^{\circ} 2$ del año 2006 un trabajo paralelo en Español en el que constan autores ya implicados en nuestros trabajos anteriores y en el que se ignora al Profesor Jara Michel, chileno, adalid de este tema en América Latina y no se haga referencia alguna al camino recorrido en Español.

Existe además una propuesta, elaborada en el Congreso Internacional de Uroginecología (IUGA) en junio de 2007 en Cancún (México), en la que también se ignora el trabajo del Grupo Español de Urodinámica y de la Sociedad Iberoamericana de Neurourología y Uroginecología.

Todos los trabajos son mejorables y con seguridad el realizado por el Grupo Español de Urodinámica y la SINUG lo es. Por ello, en un grupo que nunca ha sido excluyente, nos cuesta entender por qué se nos ignora. Pensamos que, obviamente, el esfuerzo se vería mejorado al aplicar las aportaciones críticas correctas de cualquier otro grupo o grupos que quisieran integrarse. 
No queda claro cuál ha sido la intención de ignorar el trabajo hecho, si es que la ha habido, o si sólo ha sido la resultante del silencio de algunos que no aclararon respecto al camino realizado, y con ello nos sitúan en una situación incómoda. Nosotros seguimos pensando que el Español es el mayor nexo de unión entre los pueblos Americanos y España y nos gustaría evitar malos entendidos.

Las publicaciones del grupo español de Urodinámica y la SINUG, entre otras, han sido:

- Castro-Díaz D, Conejero-Sugrañes J, MartínezAgulló E. Propuesta de terminología de la función del tracto urinario y vaciado vesical: 2. Clasificación de la disfunción del tracto urinario inferior. Urodinámica Aplicada. 1989;3(2):65-68.

- Castro-Díaz D, Martínez-Agulló E, ConejeroSugrañes J. Propuesta de terminología de la función del tracto urinario y vaciado vesical: 1. Fase de llenado y vaciado vesical. Urodinámica Aplicada. 1989;3(1):39-44.

- Castro-Díaz D, Martínez-Agulló E, Conejero-Sugrañes J. Propuesta terminológica de la función del tracto urinario. In: Martínez-Agulló E, editor. Incontinencia Urinaria. Conceptos actuales. $1^{\mathrm{a}}$ ed. Alzira (Valencia): Graficuatre; 1990. p. 737-754.

- Conejero-Sugrañes J, Martínez-Agulló E, Garriga i Calatayud J. Definición, conceptos y terminología. In: Ministerio de Sanidad y Consumo. Dirección General de Farmacia y Productos Sanitarios, editor. Libro blanco sobre la incontinencia urinaria en España. Madrid: Ministerio de Sanidad y Consumo; 1991. p. 7-14.
- Castro-Díaz D, Martínez-Agulló E, Conejero-Sugrañes J. Terminología de la función del tracto urinario: llenado. Urodinámica Aplicada. 1999; 12(1): 43-48.

- Castro-Díaz D, Conejero-Sugrañes J, MartínezAgulló E. Clasificación de la disfunción del tracto urinario inferior. Urodinámica Aplicada. 1999;12 (1):49-53.

- Grupo Español de Urodinámica y de SINUG. Estandarización de la terminología de la función del tracto urinario inferior: informe de estandarización del sub-comité de la Sociedad Internacional de Continencia. Propuestas de traducción y homologación conceptual. Urodinámica Aplicada. 2002; 15(4):283-298.

- Jara Michael O. Terminología en urodinámia. Urodinámica Aplicada. 2003;16(2):63-65.

- Grupo Español de Urodinámica y de SINUG. Consenso sobre terminología y conceptos de la función del tracto urinario inferior. Actas Urol Esp. 2005; 29(1):16-30.

- Martínez-Agulló E. Terminología de la función del tracto urinario inferior. Actas Urol Esp. 2005;29 (1):5-7.

Grupo Español de Urodinámica y SINUG.

Correspondencia autor: Dr. R. Martínez García Consenso de la SINUG y del ICCS

E-mail autor: roberto.martinez.garcia@gmail.com Información artículo: Editorial 